\title{
Self-Governing WSN'S Assumptions and its Applications
}

\author{
Ahmed Al-Utaibi \\ College of Computer Sciences and Information Technology \\ Majmah University, Saudi Arabia
}

\begin{abstract}
Up-to-date progress and developments in low-power and lowcost wireless communication tied together with advancement in ad-hoc networking routing and protocols have fashioned WSN'S a hot theme of vigorous attention. Usually, hardware modules in a WSN comprise analog-to-digital converters, sensor circuits, wireless communications transceivers and microprocessors. These hardware modules/components ought to be planned utilizing software tools to maneuver agreeably on the way to bring about a user-defined job. The major goals of such a network are data acquirement and Radio Frequency transmission. In this research article, we bring in indispensable impressions in this promising multidisciplinary research area. Two categories of WSN's applications in reallife are acknowledged. In regards to on-going exertion, a constructed and an all-inclusive devise framework of a smart wireless patient-monitoring system has been illustrated. Our preliminary purpose in this research article is probable to set off prospect research projects with a possible sketch for future work as well.
\end{abstract}

\section{Keywords}

Autonomous WSN's, Sensor Networks, Communications, Health Monitoring Sensor

\section{INTRODUCTION}

Latest progress in the IT industry en route for a novel wireless communication devices and systems and their exploitation in addressing an extensive assortment of real-world defies has reported in a number of innovative fields of lively research WSN's [Xbow.com][Berkeley's website][ACM Conference, 2003][ ACM, 2002][intel.com][ bwrc.eecs.berkeley.edu] being one such blistering area. By knowing that, the Internet has been talented to endow with an outsized number of customers with the aptitude to shift miscellaneous figures/forms of information willingly and hence transformed business, education, defense, science and industry research. Sensor networking [S.N. Simić, 2003][A. Mainwaring et al, 2002][intel.com][A.N. Knaian, 2000][W.W.Manges et al][G. Kantor et al, 2003][A. Sinha et al][L. Szumel et al][ S.D. Servetto][D. Ganesan et al] may possibly in the long term, be uniformly considerable by endowing with dimension of the physical occurrence in our surroundings, directing towards their indulgent and eventually the deployment of such information for an extensive variety of realistic applications. Prospective applications of sensor networking comprise defense/military, environmental/ecological [S.N. Simić] and habitat/environment monitoring [A. Mainwaring et al], healthcare/patient monitoring [intel.com], transportation [A.N. Knaian], manufacturing/industrial [W.W. Manges et al], and explore and salvage/rescue [G. Kantor et al].

\section{EXPLAINATION}

A usual WSN comprises of more than a few nodes disseminated or located in the milieu of attention and a base station as well. Each node is projected to identify events of interest/concentration and approximate parameters or strictures that differentiate such events. The ensuing information at such a node ought to be passing on to the base station either unswervingly or either in a "multi-hop" manner concerning involuntary routing all the way through several other nodes in the network. Execution or realization of such a network entails hardware components/modules and equivalent software modules to line up these components/modules in an accommodating approach. A profitable hardware platform that has been under investigation comprises of processor cum radio boards generally named as "motes". Each mote, a battery-powered device/tool, comprises a sensor component, a power component, a two-way ISM band radio transceiver unit or component (contains a Radio Frequency antenna), an Analogue to Digital Converter unit, a processor that runs or carries TinyOS-based code, and logger memory competent of accumulating up to 100,000 measurements/assessments. A base station comprise of a mote affixed to a mote-interfaceboard that is edged/interfaced to a Personal Computer passing through the parallel port. There are two sorts of motes in this viable system [xbow.com], explicitly; mica2 and mica2dot. Both are illustrated in Figure 1. Embraced in the artifact are so called sensor boards that unswervingly friendly with a mote. These boards take in of sensor modules/components e.g. magnetometer, accelerometer, microphone, thermistor, photosensor, and furthermore, allocate incorporation of clients individual sensors.

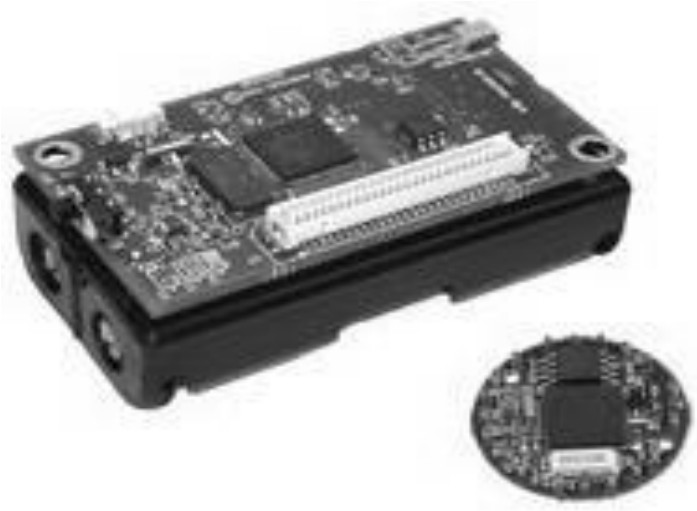

(i) 


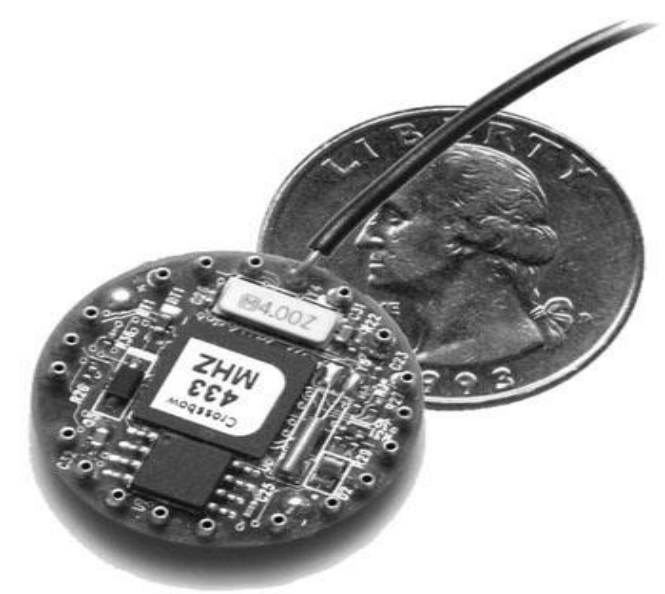

(ii)

Figure 1: Mica2dot

Figure 1: (i) Mica2dot and mica2 motes provided by Crossbow Technologies Inc. (ii) As depicted, mica2dot is as good as to a loonie coin in provisions of size. In provisions of software, the operating system (O.S) for programming this meticulous sensor network is called TINYOS [2]. TinyOS is an event-driven O.S planned for sensor network nodes having inadequate resources such as $8 \mathrm{~K}$ bytes of program memory and 512 bytes of RAM. Programming language that is utilized to have power over and to make the hardware components functional to carry out user-defined errands is called nesC. It is an expansion of $\mathrm{C}$ intended to exemplify the structuring perceptions and implementation sculpt of TinyOS. Software is written up and Berkley propped it up. Transmission and Reception is offered at unusual frequencies. Specialized mote development kits vacant in the marketplace consent to frequencies of $315 \mathrm{MHz}, 433 \mathrm{MHz}$ and $868 / 916 \mathrm{MHz}$ with distinctive exposure of $1000 \mathrm{ft}, 1000 \mathrm{ft}$ and $500 \mathrm{ft}$ correspondingly. An external battery is used to power up each mote. Power administration in WSN's [A. Sinha et al] is an imperative facet that will impel research in the subsequently decade and Radio Frequency transceivers and low-power sensors are essential for major applications in this particular field. The high-tech in power administration engrosses significant "wake" and "sleep" cycles. A mote illustrates mA current altitude in data-gathering or wake mode and only draws $\mu \mathrm{A}$ current levels in sleep mode. A variety of other realistic facets comprising practicability revisions [L. Szumel et al] [S.D. Servetto] are being practiced.

\section{CLASSIFICATION}

As the technology grows on and attains attractiveness, research is flattering imperative in both hypothetical and application perspective [ACM, 2003][ ACM, 2004]. Therefore we make out following two classes of application instances.

\subsection{Stationary or Immobile Network}

It is described as a network of sensor nodes, in which, each sensor node's location is predetermined comparative to the base station and additional nodes within the network. An established application in this regards is vineyard moisture monitoring [intel.com]. Data attained by a mote is propelled to the base station which then onwards routes the information and elicits obligatory exploits e.g. localized watering. There are two potential cases to put out data. When a node is in undeviating wireless is in the range of the base station, unswerving communication is achievable. When a node is not in the coverage, it puts on the air the data in an ad-hoc setting also passed on to as multi-hop. Realization of a wellorganized multi-hop system has a need of an efficient routing [D. Ganesan et al] to smooth the progress of shortest route, abridged power utilization and enhanced communication.

\subsection{Network in Motion (Movable or Mobile)}

This class can be illustrated by an example of a flock of animals on a widespread cattle farm, where all animals are equipped or outfitted with a sensor node. The animals are in an invariable movement comparative to the base station and to each other as well. Such complex mobility supervision entails a constant and more refined realization of routing algorithms. In order to attain more assistance from wireless sensor networks of this category, we anticipate further hardware rations in the shape of GPS devices and also by means of mote position.

\section{WIRELESS SENSOR SYSTEMS BASED PATIENT MONITORING}

As fraction of the enduring exertion, an inclusive design framework of an intellectual wireless patient-monitoring system has been proposed. Which comprises synchronized sensing of patient's fundamental parameters/dimensions utilizing the motes, and wireless communication of such decisive information over R.F to the base-station. Ensuing data processing on a Personal Computer will consent to uncovering of convinced medical urgent situations, and involuntary alerts of medical team. The entire framework is exposed in Figure 2.

In Projects stage I, a cut down archetype system bringing into play an indispensable wireless network kit and sensor components has been incorporated just because of experimentation. In next phase which is phase II of the project, biomedical leaning high-precision and low-power sensors will be premeditated and prospects of mounting or structuring a digital (Bluetooth) description of the WSN will be considered for progression of the archetype. And this system will be experienced in a hospital or medical setting and will be distinguished or advanced in regards of meeting ethical/moral, technological and medical principles in subsequent phase of the project. Prospective deliverable will take in:

i. An Ad-Hoc self-administering wireless system is competent enough of secure patient-monitoring still when patients are in action.

ii. An improved automation of Smart-Bed in provisos of urgent situation revealing.

These qualifications and aptitude could show the way to a well-organized utilization of a priceless healthcare possessions. The description of system maneuver, together with its significance to the "TRLabs Home Technologies initiative" has been proposed. It comprises a synchronized manifestation/display of a wireless self-organizing sensor network concerning routine sensors. 


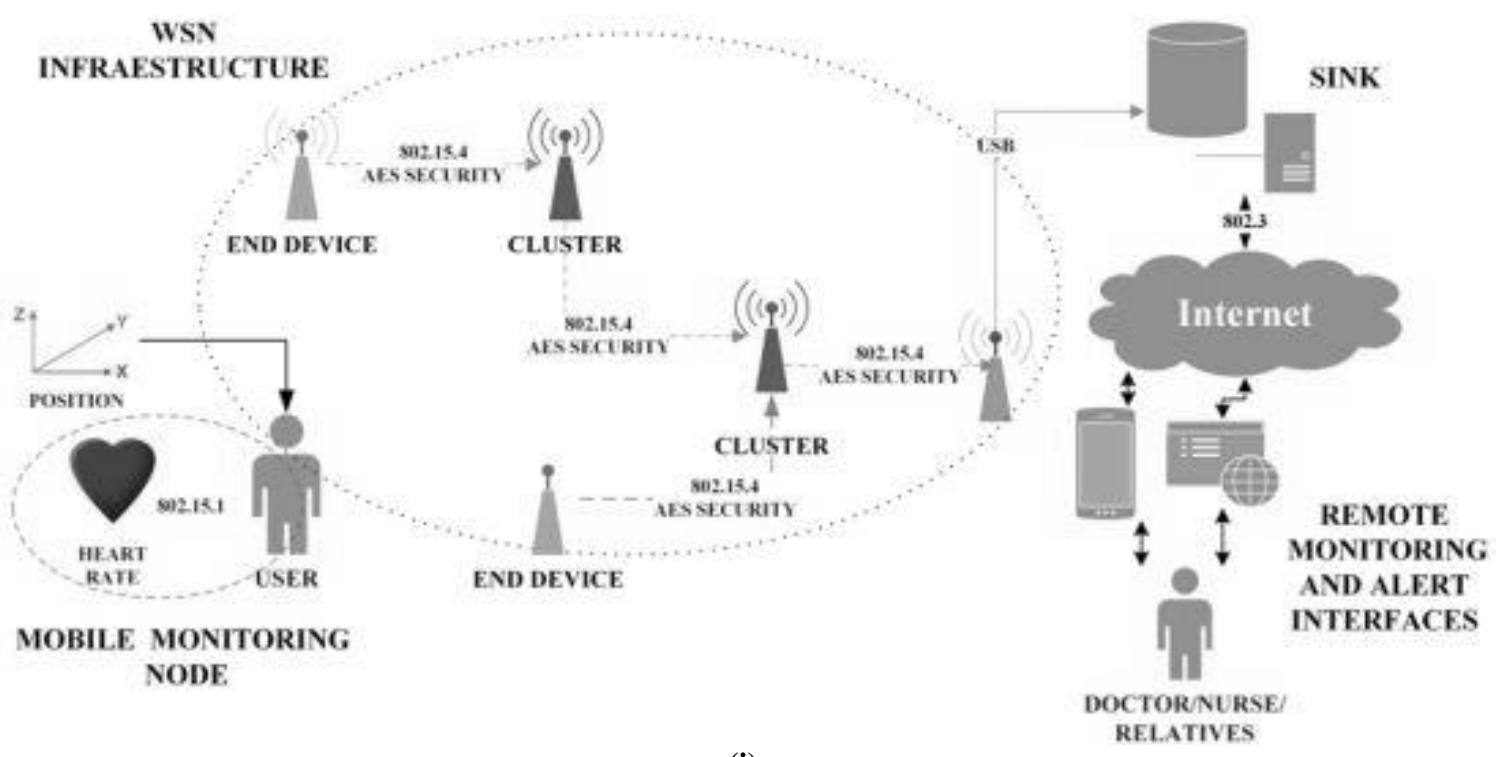

(i)

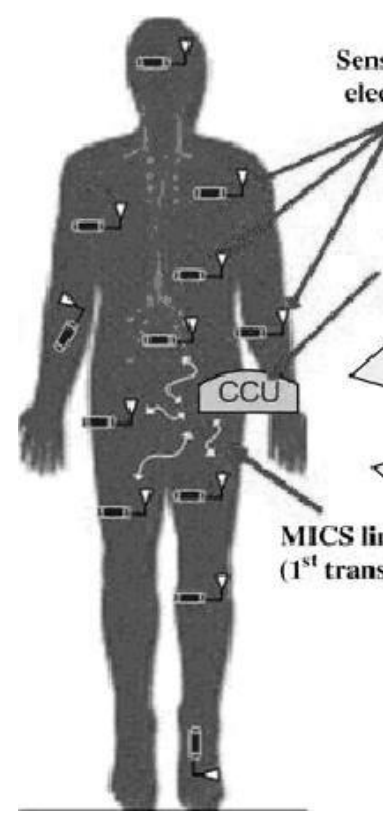

Web-based

Internet trans.

REL.ATIVES

MONITORING

AND ALERT

INTERFACES

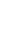

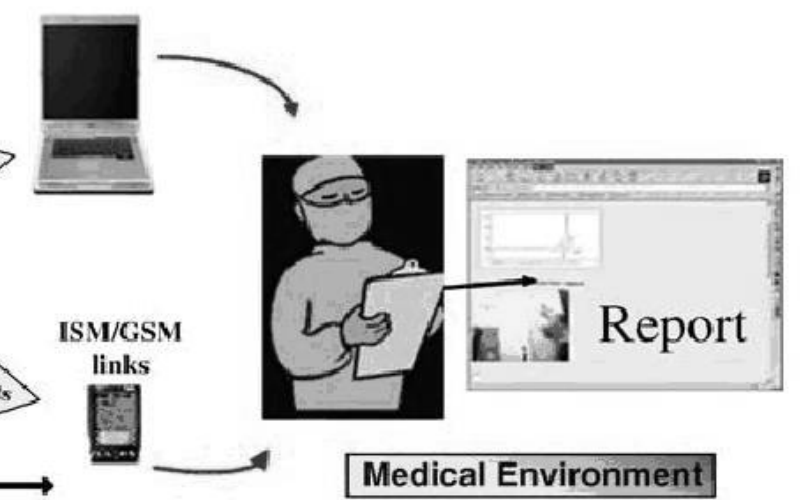

Long distance information transmission ( $2^{\text {nd }}$ transmission link)

(ii)

Figure 2: (i, ii) Medical Emergencies Detection and Alerting System

\section{CONCLUSIONS}

In IT a new-fangled field, that is to say wireless selforganizing, adaptable, flexible sensor networks, is launched. Hardware and software $(\mathrm{H} / \mathrm{W} \& \mathrm{~S} / \mathrm{W})$ components/modules are in brief illustrated. Application paradigm is off the record into stationary/immobile networks and networks in motion/action. An innovative application in healthcare or medical zone is anticipated or projected and an archetype has put into practice for notion expression. This novel field pledges networking of the milieu nearby us. An appealing facet or feature is that this research area takes in collectively an assortment of electrical engineering fields comprising squat power devise, Radio Frequency/wireless communications, signal processing, networking and optimization.

\section{REFERENCES}

[1] http://www.xbow.com,
[2] http://today.cs.berkeley.edu/tos/,

[3] http://www.intel.com/labs/features/rs01031.htm.

[4] http://bwrc.eecs.berkeley.edu/,

[5] http://www.intel.com/research/exploratory/wireless_pro mise.htm\#design,

[6] "S.D. Servetto", "On the feasibility of large-scale wireless sensor networks," 40th Annual Allerton Conf. on Communication, Control and Computing, Urbana, IL, October 2002.

[7] "S.N. Simić", "Distributed environmental monitoring using random sensor networks," 2nd Int. Workshop on Information Processing in Sensor Networks at the Palo Alto Research Center, Palo Alto, CA, April 2003

[8] ACM Conference on Embedded Networked Sensor Systems, Los Angeles, CA, November 2003. 
[9] "W.W. Manges et al", "Intelligent wireless sensors for industrial manufacturing," Web Document, Oak Ridge National Laboratory.

[10] "L. Szumel et al", "On the Feasibility of the UC Davis Metanet," Technical Report ECE-CE-2003-2, Computer Engineering Research Laboratory, University of California, Davis, 2003.

[11] "D. Ganesan et al", "Highly resilient, energy efficient multipath routing in wireless sensor networks," Mobile Computing and Communications Review, vol. 1, no. 2, 2002.

[12] "A.N. Knaian", "A wireless sensor network for smart roadbeds and intelligent transportation systems," M.Eng. Thesis, Department of Electrical Engineering and Computer Science, Massachusetts Institute of Technology, June 2000.
[13] "G. Kantor et al", "Distributed search and rescue with robot and sensor teams, Proc. 4th Int. Conf. on Field and Service Robotics, Lake Yamanaka, Japan, July 2003.

[14] ACM International Workshop on Sensor Networks and Applications, Atlanta, GA, September 2002.

[15] "A. Sinha et al", "Dynamic Power Management in Wireless Sensor Networks", IEEE Design and Test of Computers, vol. 18, no. 2, 2001.

[16] "A. Mainwaring et al", "Wireless sensor networks for habitat monitoring," ACM International Workshop on Sensor Networks and Applications, Atlanta, GA, September 2002. 